\title{
The role of carbon nanotubes in both physical and chemical liquid-solid transition
}

\section{of polydimethylsiloxane}

L.J. Romasanta*, M.A. Lopez Manchado and R. Verdejo*

Instituto de Ciencia y Tecnología de Polímeros ICTP - CSIC, Juan de la Cierva 3, 28006 Madrid, Spain

*E-mail: rverdejo@ictp.csic.es; laura.jimenez@ictp.csic.es; Fax: +34 91564 4853; Tel: +34915622900

\begin{abstract}
In this work we investigate the effect of the dispersion degree of as-received multiwall carbon nanotubes (MWNTs) in the liquid-solid transitions (LST) of polydimethylsiloxane (PDMS). The physical LST of the suspensions showed a strong dependence on the nanotubes dispersion degree due to the formation of an interconnected network as the dispersion time increased. These MWNTs acting as "effective physical cross-links" also influenced the dynamic viscoelastic properties, accelerating the chemical LST (or chemical cross-linking) and giving rise to an increase in the cross-linking density of the composite samples.
\end{abstract}

Keywords: carbon nanotubes; polydimethylsiloxane; cross-linking reaction; rheological properties; dispersion

\section{Introduction}

During the past decade, the liquid-solid transition (LST) in polymer materials have been extensively explored since it is a crucial technological point delimiting their processing conditions, properties and applicability [1]. Independently of the nature of the LST, i.e. physical or chemical, this phenomenon represents a change in the 
molecular mobility of the polymer matrix and can hence be easily followed by rheological measurements. One of the first systems to be analysed by rheology was a polydimethylsiloxane (PDMS), that presents a LST due to the chemical cross-linking of its chains and provides ideal model networks [2]. Furthermore, the excellent chemical and thermal properties of PDMS make it a widely used elastomer in commercial applications. However, it requires the addition of fillers in order to get a substantial improvement of its modulus and strength. The most common fillers added to PDMS are silica and titanium dioxide particles which have shown to provide a good mechanical reinforcement [3].

Current scientific interest on the use of nanofillers, and in particular carbon nanotubes, has been moderately investigated with PDMS showing mechanical reinforcement and increases in electrical conductivity [4-7]. The key-point for obtaining these enhanced properties stems from an effective dispersion and formation of a MWNT physical network in the PDMS polymer matrix prior and after its chemical crosslinking. Therefore, to attain this interpenetrating structure between filler and polymer, it is necessary to break apart the MWNT bundles and to stabilise the individual nanotubes in order to prevent their re-aggregation [8].

The use of rheological measurements has already been reported as an efficient method to understand the nanofiller dispersion degree [9-11]. A direct consequence of a homogeneous dispersion of MWNTs in a polymer matrix is the significant change in the viscoelastic properties [12-16]. Since the rheological parameters of a (nano)composite are correlated with the distribution of (nano)fillers within the polymer measuring $G^{\prime}$, $G^{\prime \prime}$ and $\eta^{*}$ at different stages of the mixing process can provide information about the time necessary to achieve a suitable level of dispersion [9]. Recent studies have analysed the linear viscoelastic behaviour and re-aggregation mechanisms of semi- 
dilute suspensions of MWNTs/PDMS prior to the cross-linking process observing the formation of an elastic nanotube network $[13,17]$. Nevertheless, and to the best of our knowledge, the effect of fillers in the cross-linking process of PDMS has been generally overlooked until very recently when Estevez et al. [18] analysed the influence of microsize garnet particles. The authors observed a slowdown of the overall cross-linking process due to a strong interaction of the particles with the catalyst. The influence of nano-size fillers and, in particular, of MWNTs with their ability to form an elastic network on the chemical LST of PDMS materials has not been investigated despite the fact that is one of the major factors that govern the ultimate properties of the resulting material. Hence, the influence of MWNT dispersion degree on the cross-linking reaction must be assessed in order to achieve and guaranty good overall performances of the resulting elastomeric composites.

In summary, this study analyses both physical and chemical liquid-solid transition of semi-dilute MWNT/PDMS suspensions by measuring the linear viscoelastic and dynamic rheological properties, respectively, of the system.

\section{Materials and Methods}

\subsection{Materials.}

Multiwall carbon nanotubes (MWCNTs Thermally Purified) were kindly supplied by FutureCarbon $\mathrm{GmbH}$. They were synthesised by chemical vapour deposition (CVD) with a mean diameter of $15 \mathrm{~nm}$, an average length ranging from 5 $50 \mu \mathrm{m}$ and a purity of $98 \%$. A commercial poly(dimethyl)siloxane based formulation (PDMS RTV4420 A/B) supplied by BlueStar Silicones, was used as polymer matrix. This room temperature curing system consists of two components: Part A composed of 
a vinyl terminated polymer $(\equiv \mathrm{SiVi})$ together with a platinum catalyst, while Part $\mathrm{B}$ contains the pre-polymer $(\equiv \mathrm{SiH})$.

\subsection{Preparation of MWNT/PDMS suspensions.}

Given that the basis of the work is on the quality of the suspensions prepared, special attention must be paid on how to prepare the aforementioned suspensions. It is well known that the most common and widely used dispersion techniques for suspensions in a low-medium viscosity material are "high-shear" mixing and ultrasonication [19]. The latter is able to release higher power and is quite efficient to separate MWNT agglomerates, but it also may cause a considerable damage in MWNT structure due to the violent cavitation $[20,21]$. To evaluate the possible breakage of the MWNTs during mixing, Huang et al. [9] calculated the shear stress applied to MWNTs mixtures as follows:

$$
\sigma \approx \frac{\eta R \omega}{h}
$$

Where $\eta$ is the polymer viscosity, $\omega$ is the angular frequency of the mixer, $R$ and $h$ are the radius and the gap of the vessel employed. They obtained a shear stress of $1 \mathrm{MPa}$, which compared to the tensile stress of single and multiwall carbon nanotubes of about 200-900 MPa [22-24], indicated a low level of nanotube breaking. Applying this analysis to our system, we obtained an applied shear stress of about $5 \mathrm{MPa}$, which is again considerably lower than the tensile stress of MWNTs. Hence, we selected the high shear mixing protocol to avoid major damages to our MWNTs. We should point out that MWNTs concentration was kept constant along this study since the aim of this work is to clarify the influence of MWNTs dispersion degree on both physical and chemical LST of the raw polymer. 
Samples of $10 \mathrm{~g}$ total weight with $0.5 \mathrm{wt} \%$ of MWNTs were prepared by directly adding the nanotubes onto $\equiv \mathrm{SiH}$ pre-polymer. The dispersion time was then chosen as the variable parameter and hence, MWNTs were mechanically mixed (Ika Mixer) at $2400 \mathrm{rpm}$ for different times $(\mathrm{t}=2,4,6$, and $24 \mathrm{~h})$. Changes in the microstructure of the suspensions were studied measuring the linear viscoelastic properties immediately after the desired dispersion time.

\subsection{Preparation of MWNT/PDMS composites.}

MWNT/PDMS cross-linked composites were obtained by mixing $\equiv \mathrm{SiVi}$ prepolymer with the suspensions previously prepared in a ratio 1:1. After stirring the mixture for 2 minutes, the pasty suspensions were degassed in a vacuum oven at 30-50 mbar for approximately 10 minutes to remove air bubbles formed during mixing operations. Films with an average thickness of about $0.5 \mathrm{~mm}$ were prepared by mould casting and then cured at room temperature for $24 \mathrm{~h}$ before swelling and microstructural characterisation.

\section{$2.4 X$-ray photoelectron spectroscopy (XPS).}

The chemical composition of MWNTs was evaluated by X-ray photoelectron spectroscopy (XPS). Photoelectron spectra were acquired with a VG Escalab 200 R spectrometer equipped with a hemispherical electron-analyser and a $\mathrm{MgK} \alpha(\mathrm{hv}=1253.6$ $\mathrm{eV}$ ) non-monochromatic X-ray source. The $\mathrm{O} 1 \mathrm{~s}$ and $\mathrm{C} 1 \mathrm{~s}$ signals were scanned using the binding energy (BE) of $\mathrm{C} 1 \mathrm{~s}$ core-level peak at $284.9 \mathrm{eV}$ as reference.

\subsection{Rheological measurements.}

The rheological properties were measured on a stress-controlled rheometer (TA Instruments Advanced Rheometer AR1000). Experiments were performed at room temperature using parallel plate geometry (gap $=500 \mu \mathrm{m})$ with a $20 \mathrm{~mm}$ upper corrugated plate. Stress sweep tests (frequency kept constant at $1 \mathrm{~Hz}$ ) were carried out to 
monitor the extent of each sample's linearity. After the sample's linear viscoelastic region was defined, dynamic oscillation frequency sweeps were performed at the terminal region $(0.01-10 \mathrm{~Hz})$ to monitor the state of the MWNT dispersion. Isothermal curing runs with constant shear frequency $(1 \mathrm{~Hz})$ in the linear viscoelastic regime were also performed to determine the influence of the cross-linking reaction on the viscoelastic properties.

\subsection{Optical and electron microscopy.}

The dispersion degree of MWNT/ $\equiv \mathrm{SiH}$ suspensions and MWNT/PDMS crosslinked samples were analysed by optical microscopy (Carl-Zeiss Jenna optical microscope equipped with a CCD-Iris Sony video camera) and scanning electron microscopy (ESEM XL30 Model, Philips), respectively.

\subsection{Swelling measurements.}

The average mass of network chains between cross-links $M_{c}$ was calculated using equilibrium-swelling measurements by applying the phantom model approximation:

$$
\ln \left(1-\emptyset_{\mathbf{r}}\right)+\emptyset_{\mathbf{r}}+\mathrm{X} \emptyset_{\mathbf{r}}=-\frac{P_{\mathrm{r}}}{\mathrm{M}_{\mathrm{c}}} \mathrm{V}_{\mathrm{s}}\left(1-\frac{2}{\mathrm{f}}\right) \emptyset_{\mathrm{r}} \frac{1}{3}
$$

Where $\Phi_{r}$ is the rubber volume fraction (properly obtained by considering that carbon nanotubes cannot be swollen), $\chi$ is the solvent-polymer parameter $(\chi=0.465$ for toluenePDMS system), $\rho_{r}$ is the rubber density (measured using the hydrostatic weighing method and applying the Archimedes principle), $V_{s}$ is the molar volume of the solvent employed $\left(V_{s}=106.2 \mathrm{ml} / \mathrm{mol}\right.$ for toluene) and $f$ is the functionality of the rubber network formed (assumed to be tetrafunctional in our case, $f=4$ ). Five weighed test pieces of each cross-linked sample were swollen in toluene at room temperature. These vulcanized samples were weighed at regular intervals (24, 48 and 72 hours), storing the vessels in a dark cabinet to avoid photodegradation reactions of the compounds. All 
samples showed a constant weight over the entire interval and, thus, diffusion equilibrium was reached after $24 \mathrm{~h}$.

\section{Results and discussion}

\subsection{Effect of carbon nanotubes dispersion degree on the physical LST.}

Fig. 1 shows the variation of the rheological parameters for $\mathrm{MWNT} / \equiv \mathrm{SiH}$ suspensions as a function of dispersion time. The suspending fluid $(\equiv \mathrm{SiH})$ follows a nearly frequency-independent quasi-Newtonian behaviour. Low-medium viscosity Newtonian polymers are particularly useful for the purpose here reported since this type of materials can impart significant shear stress to MWNT bundles without masking their viscoelastic response[25].

The addition of MWNT leads to a gradual increase of the viscosity of the suspension and a shear thinning behaviour where the viscosity decreases with increasing frequency, being this last fact a clear indication of a solid-like behaviour. The change from liquid-like to solid-like behaviour due to the formation of filler-filler and fillerpolymer networks as a result of the dispersion time is clearly noticed in Fig.1 . The sample stirred for 24 hours shows an increase in the absolute complex viscosity $\eta^{*}$ up to four orders of magnitude in relation to the unfilled sample together with a marked shear thinning behaviour. The $6 \mathrm{~h}$ suspension shows similar viscoelastic properties to the $4 \mathrm{~h}$ suspension and hence, for the sake of clarity, the corresponding results will be omitted.

\section{Insert Fig.1}

It is generally accepted that highly asymmetric nanoparticles are more effective in changing the properties of the polymeric matrix than spherical or sheet-like nanoparticles [26]. Rod-like nanoparticles, in this case MWNTs, can easily form 
network structures either through direct interaction among each other or polymeric chain bridging between at least two different nanoparticles. To analyse this change in the suspension microstructures, storage (elastic) modulus $G$ ' and loss (viscous) modulus $G$ " of the unfilled and filled $\equiv \mathrm{SiH}$ are also displayed in Fig.1. The polymeric matrix shows the typical terminal regime with values of $G^{\prime} \sim \omega^{2}$ at low frequencies. However, for $\mathrm{MWNT} / \equiv \mathrm{SiH}$ suspensions, the lack of sensitivity of $G^{\prime}$ to low frequencies indicates that the nanoparticle dispersion is enhanced with dispersion time, as clearly observed in the optical micrographs of the suspensions (Fig.2). Thus, the suspensions exhibit a more homogenous morphology with smaller agglomerates for higher dispersion times. This observation is further corroborated with a change in the viscoelastic response from the physical liquid-solid transition between 2 and $4 \mathrm{~h}$ of dispersion. The formation of a nanotube network plays a significant role in this transition, giving rise to additional physical cross-links and large contributions to the suspensions viscoelasticity, i.e. $G$ ' increases several orders of magnitude than raw polymer as the dispersion time increases. The well-known frequency-independence of $\tan (\delta)$ for cross-linked systems [27] can also be applied to determine the exact point at which the physical LST takes place. This point can easily be determined from the cross of a multifrequency semilogarithmic plot of $\tan (\delta)$ versus the time of dispersion used.

\section{Insert Fig. 2}

\section{Insert Fig. 3}

Results shown in Fig.3 indicate that, for our system, the "physical gelation" time $\left(t_{\mathrm{pg}}\right)$ is achieved after approximately $\mathrm{t}_{\mathrm{pg}}: 200$ min of dispersion, which means that:

I) For low dispersion times $\left(\mathrm{t}<\mathrm{t}_{\mathrm{pg}}\right) G^{\prime}$ remains lower than $G$ ', whatever the frequency. Most of the energy of deformation is dissipated as heat due to 
increased friction between MWNTs and $\equiv \mathrm{SiH}$ pre-polymer as a result of the weak interaction between both components while,

II) For high dispersion times $\left(\mathrm{t} \geq \mathrm{t}_{\mathrm{pg}}\right)$ an optimised dispersion of the MWNTs alters the terminal region due to the restricted movement of the polymeric chains. The storage modulus starts to develop a rubbery plateau at low frequencies, which is a typical rheological feature of physical gelation since the nanotubes begin to act as "effective physical cross-links" and are capable of connecting the polymer in a large scale structure. Hence, once an interpenetrating structure is generated between the filler and the polymer matrix, the system acts as an elastic solid at low frequencies. An illustrative picture of the effect of MWNT dispersion as a function of the dispersion time is shown in Fig.4.

\section{Insert Fig. 4}

\subsection{Effect of carbon nanotubes dispersion degree on the chemical LST.}

$\mathrm{MWNT} / \equiv \mathrm{SiH}$ suspensions were mixed with the $\equiv \mathrm{SiVi}$ pre-polymer obtaining an elastomeric network, via hydrosilylation reaction, where the MWNTs were entrapped. The uniform distribution of MWNTs in the elastomeric matrix (Fig.5) for the composite at the longest dispersion time evidences that an increase of the dispersion time is an effective approach to separate the MWNTs aggregates/bundles.

\section{Insert Fig. 5}


The chemical LST (or cross-linking reaction) that takes place relies on the ability of the hydrosilane bond of the cross-linker $(\equiv \mathrm{SiH})$ to add across a carbon-carbon double bond that belongs to the pre-polymer $(\equiv \mathrm{SiVi})$ in the presence of Pt catalyst.

$$
\equiv \mathrm{Si}-\mathrm{CH}=\mathrm{CH}_{2}+\mathrm{H}-\mathrm{Si} \equiv \stackrel{\mathrm{Pt}}{\rightarrow} \equiv \mathrm{Si}-\mathrm{CH}_{2}-\mathrm{CH}_{2}-\mathrm{Si} \equiv
$$

This chemical process proceeds simultaneously to secondary reactions due to the reaction of silane groups $(\equiv \mathrm{Si}-\mathrm{H})$ with atmospheric moisture and $\mathrm{O}_{2}$ (reactions (ii) and (iii)) in the presence of temperature and the catalyst. These secondary reactions lead to the formation of silanol groups $(\equiv \mathrm{Si}-\mathrm{OH})$ which can catalytically react with remaining $\equiv \mathrm{Si}-\mathrm{H}$ groups (reaction (iv)) or even with other silanol groups (reaction (v)) to produce silsesquioxane structures or additional $\equiv \mathrm{Si}-\mathrm{O}-\mathrm{Si} \equiv$ cross-links $[28,29]$. Nevertheless, these secondary reactions are slower than the hydrosilylation reaction and only relevant at very high temperatures [30].

$$
\begin{aligned}
& \equiv \mathbf{S i}-\mathbf{H}+\mathbf{H}_{2} \mathbf{O} \stackrel{\mathrm{Pt}_{3} \mathrm{~T}}{\rightarrow} \equiv \mathrm{Si}-\mathbf{O H}+\mathbf{H}_{2} \\
& 2 \equiv \mathrm{Si}-\mathrm{H}+\mathrm{O}_{2} \stackrel{\mathrm{Pt}_{3} \mathrm{~T}}{\rightarrow} 2 \equiv \mathrm{Si}-0 \\
& \equiv \mathbf{S i}-\mathbf{H}+\mathbf{H O}-\mathrm{SiH} \equiv \stackrel{\mathrm{Pt}_{\mathbf{T}} \mathbf{T}}{\longrightarrow} \equiv \mathbf{S i}-\mathbf{O}-\mathbf{S i} \equiv+\mathbf{H}_{2} \\
& \equiv \mathrm{Si}-\mathrm{OH}+\mathrm{HO}-\mathrm{SiH} \equiv \stackrel{\mathrm{Pt}_{\mathrm{t}} \mathrm{T}}{\longrightarrow} \equiv \mathrm{Si}-\mathbf{O}-\mathrm{Si} \equiv
\end{aligned}
$$

Hydrosilylation reactions catalyzed by a platinum complex exhibit often an autocatalytic "S" shape with a gel-point near the inflection point defined as the moment at which an infinite network is first formed (Fig.6).

\section{Insert Fig. 6}


The chemical LST is a continuous process where three main stages can clearly be observed [30, 31]:

I) Induction period, where the viscous effect dominates the process of crosslinking. This region indicates the safe processing time since $G$ ' value increases rapidly due to its sensitive to the structure change (gel formation).

II) Curing reaction period, where the cross-linking network starts to form and the stiffness of the elastomer increases. Most of the final product is produced in a relatively small fraction of the total reaction time.

III) Post-cure period, where the cross-linking reaction stops. During this stage, the values of $G^{\prime}$ and $G$ '" establish the final properties of the elastomeric matrix formed although some additional but slower cross-linking through secondary reactions may occur.

Nevertheless, prior to the analysis of the effect of MWNT network previously formed on the chemical LST, we should elucidate possible interactions between the Pt catalyst and the MWNTs as a result of potential MWNT surface functionalities. Several authors have reported that the presence of polar functionalities (namely carboxylic and/or alcohols groups) offers possible binding sites for complexation of metallic Pt. This fact can lead, in turn, to the formation of complexes that are stable at room temperature with the subsequent inhibition/decrease of the cross-linking reaction [18, 32]. XPS measurements (Fig.7) of the MWNTs used in this work reveal an oxygen content of $0.7 \%$ due to chemisorption during or after the original synthesis. The value is typical of a range of as-produced CVD nanotubes and confirms a relatively low content of functional groups covalently bonded at the end terminal and/or sidewalls. Hence, 
possible interactions between the nanotubes and the Pt catalyst during the cross-linking reaction can safely be neglected.

\section{Insert Fig.7}

The evolution of the chemical LST is shown in Fig.8. It is generally accepted that the equilibrium storage modulus $G^{\prime}$ value is proportional to the cross-linking network being formed by both chemical bonds and physical entanglements. At time zero, the values of $G^{\prime}$ for the filled suspensions are all higher than for unfilled sample, thus evidencing the MWNTs act as "effective physical cross-links". All samples, except the $24 \mathrm{~h}$ MWNT dispersion, clearly present the three stages described above. Meanwhile, the $24 \mathrm{~h}$ MWNT/PDMS sample shows no induction period and we observe a regular acceleration of the cross-linking reaction and a decrease of the time required to reach the plateau regimen. This effect is even clearer at long dispersion times, i.e. the longer the dispersion time the earlier the crossover of $G^{\prime}$ and $G^{\prime \prime}$ appears. Thus, the presence of the MWNT physical network appears to positively contribute to the formation of the chemical PDMS network.

\section{Insert Fig. 8}

A characteristic feature of rheological studies of cross-linking process is the rheological conversion degree $\beta$, which reflects the kinetics of the growing cross-links over time. This parameter can be defined on the basis of the change of the storage modulus $G$ ' as follows:

$$
\beta=\frac{G^{\prime}(t)-G^{\prime}(0)}{G^{y}(\infty)-G^{y}(0)}
$$


where $G^{\prime}(0), G^{\prime}(\infty)$ and $G^{\prime}(t)$ represent the initial, final and current values of the storage modulus, respectively, during the chemical LST. Rheo-kinetic models based on the evolution of $\beta v s t$ enable to follow the entire process of network formation. To monitor the cross-linking process in heterogeneous reactive systems, Malkin and Kulichikhin [33] have proposed a general equation which contains a self-accelerating term:

$$
\frac{d \beta}{d t}=k(1+c \beta)(1-\beta)^{n}
$$

where $\beta$ is the rheological conversion degree at time $t, k$ is the constant of the initial reaction rate, $c$ is the self-accelerating term, and $n$ is the reaction order. This rheokinetic model has been applied to analyse the cross-linking behaviour of a wide variety of thermoset materials, such as epoxy, melaminoformaldehyde and carbamide resins, and low molecular silico-organic elastomers [33]. Although Eq. 4 should only agree with the experimental data after the point of gel formation, the results given above confirm its validity throughout the entire chemical LST. The integration of Eq. 4 for a first and second order reaction is respectively:

$$
\begin{aligned}
& \frac{\ln (1+a \beta)}{1-\beta}=(1+c) k t \\
& \frac{\beta}{1-\beta}+\frac{c}{1+c} \ln \frac{1+c \beta}{1-\beta}=(1+c) k t
\end{aligned}
$$

The experimental and theoretical dependencies of the rheological conversion degree with the time are compared in Fig.9.

\section{Insert Fig. 9}


The viability of fitting the experimental data to a first-order rheo-kinetic expression is confirmed by the correlation coefficients $r^{2}$ shown in Table 1. The constant $k$ of the initial reaction rate has a tendency to regularly increase with increasing MWNT dispersion time, while the self-accelerating term $c$ does not show any apparent dependency on MWNT dispersion degree and is only affected by its presence. This result supports the proposed self-acceleration character of the process as a consequence of both the chemical LST and physical changes, being the latter a consequence of the enhanced MWNT network formed.

\section{Insert Table 1}

The presence of well-dispersed MWNTs and the associated initial "solid-like" behaviour results, somehow, in a regular acceleration of the cross-linking process and thus, a more rapid approach to both the $G^{\prime}, G^{\prime}$ ' crossover point and the post-cure period. This fact is quite surprising if we consider the high values of viscosity for the initial suspensions. The change in the physical properties (namely complex viscosity $\eta^{*}$ ) could affect the diffusion of the reactants during the chemical LST and, therefore, disrupt the cross-linking reaction decreasing its conversion degree. The very high aspect ratio of nanoparticles can lead to a vast interfacial area between nanotubes and the host polymer, thus reducing the macromolecular chain mobility in the vicinity of the nanotubes for several radius of gyration [34]. Nevertheless, in this case, the physical network formed in the initial suspensions influences the viscoelastic properties of the final silicone network. Results in Table 2 show that $G$ ' value at the post-cure regimen gradually increases as increasing the dispersion time due to the presence of isolated MWNTs acting as "effective physical cross-links". 


\section{Insert Table 2}

The increase of the $G^{\prime}$ value is an indicative of the increasing cross-linking density of the nanocomposites. The swelling measurements are consistent with the observed viscoelastic behaviour of the cross-linked samples, i.e. the longer the dispersion time used for the MWNT suspension, the lower the swelling degree. Such behaviour can be explained by the decrease in the molecular weight between cross-links $M_{c}$ due to the MWNT "effective physical cross-links", which reduce the length between the chemical cross-links, lower the swelling degree of the elastomeric matrix and enhances the elastic resistance to the swelling stress.

\section{CONCLUSIONS}

The objective of the present work was to study how MWNT dispersion degree could affect both the physical and chemical LST of a given polydimethylsiloxane.

Experiments have shown that, for a given mixing conditions, there is a critical time $\left(\mathrm{t}_{\mathrm{pg}}\right)$ necessary to achieve a physical LST. This apparent formation of a MWNT network interpenetrating the polymer matrix rose the rheological parameters $\left(\eta^{*}, G^{\prime}\right.$ and $\left.G^{\prime \prime}\right)$ to values several orders of magnitude greater than those observed for the raw polymer. The chemical LST of the MWNT/PDMS samples is well described by a first order rheo-kinetic equation, which takes into account the self-acceleration effect that arises from the chemical reaction and the micro-structural changes due to both MWNT presence and dispersion degree. The chemical cross-linked networks were formed faster and the induction stage was achieved earlier with MWNT dispersion degree. Increasing 
MWNT dispersion time also lead to a significant decrease of $M_{c}$ values, thus, attesting the implication of MWNT dispersion degree in the cross-linking process.

This work demonstrates that the large aspect ratio of MWNTs allows them to be considered as extra molecular chains that may provide additional sources of entanglements or physical cross-link in cross-linked networks and hence, increase the overall cross-linking degree of elastomers.

\section{Acknowledgments}

The authors gratefully acknowledge the financial support of the Spanish Ministry of Science and Innovation (MICINN) through project MAT 2010-18749 and the 7th Framework Program of E.U. through HARCANA (NMP3-LA-2008-213277). The authors would like to thank Dr. José Luis García Fierro for XPS measurements.

\section{References}

[1] Winter H, Mours M. Rheology of Polymers Near Liquid-Solid Transitions. Adv Polym Sci. 1997;134:165-234.

[2] Chambon F, Winter HH. Analysis of linear viscoelasticity of a crosslinking polymer at the gel point. J Rheol. 1986;30(2):367-382.

[3] Paul DR, Mark JE. Fillers for polysiloxane ("silicone") elastomers. Prog Polym Sci. 2010;35:893-901.

[4] $\mathrm{Hu} \mathrm{CH}$, Liu $\mathrm{CH}$, Chen LZ, Fan SS. Semiconductor behaviors of low loading multiwall carbon nanotube/poly(dimethylsiloxane) composites. Appl Phys Lett. 2009;95(10):103103. 
[5] Paul J, Sindhu S, Nurmawati MH, Valiyaveettil S. Mechanics of prestressed polydimethylsiloxane-carbon nanotube composite. Appl Phys Lett. 2006;89(18):184101.

[6] Chua TP, Mariatti M, Azizan A, Rashid AA. Effects of surface-functionalized multiwalled carbon nanotubes on the properties of poly(dimethyl siloxane) nanocomposites. Compos Sci Technol. 2010;70(4):671-677.

[7] Dyke CA, Tour JM. Covalent Functionalization of Single-Walled Carbon Nanotubes for Materials Applications. J Phys Chem A. 2004;108(51):11151-11159.

[8] Fan Z, Advani SG. Rheology of multiwall carbon nanotube suspensions. J Rheol. 2007;51(4):585-604.

[9] Huang YY, Ahir SV, Terentjev EM. Dispersion rheology of carbon nanotubes in a polymer matrix. Phys Rev B: Condens Matter. 2006;73(12):125422.

[10] Cassagnau P, Barrès C. Rheological Behavior of Rubber Nanocomposites. Rubber Nanocomposites. Singapore: John Wiley \& Sons; 2010. p. 353-390.

[11] Tomohiro Y, Schulz SC, Buschhorn ST, Schulte K. Investigation of shear thinning behavior and microstructures of MWCNT/epoxy and CNF/epoxy suspensions under steady shear conditions. Eur Polym J. 2012;48(6):1042-1049.

[12] Hobbie EK, Fry DJ. Nonequilibrium Phase Diagram of Sticky Nanotube Suspensions. Phys Rev Lett. 2006;97(3):036101.

[13] Moreira L, Fulchiron R, Seytre Gr, Dubois P, Cassagnau P. Aggregation of Carbon Nanotubes in Semidilute Suspension. Macromolecules. 2010;43(3):1467-1472.

[14] Schulz SC, Faiella G, Buschhorn ST, Prado LASA, Giordano M, Schulte K, et al. Combined electrical and rheological properties of shear induced multiwall carbon nanotube agglomerates in epoxy suspensions. Eur Polym J. 2011;47(11):2069-2077. 
[15] Rahatekar SS, Koziol KKK, Butler SA, Elliott JA, Shaffer MSP, Mackley MR, et al. Optical microstructure and viscosity enhancement for an epoxy resin matrix containing multiwall carbon nanotubes. J Rheol. 2006;50(5):599-610.

[16] McClory C, McNally T, Baxendale M, Pötschke P, Blau W, Ruether M. Electrical and rheological percolation of PMMA/MWCNT nanocomposites as a function of CNT geometry and functionality. Eur Polym J. 2010;46(5):854-868.

[17] Marceau S, Dubois P, Fulchiron R, Cassagnau P. Viscoelasticity of Brownian Carbon Nanotubes in PDMS Semidilute Regime. Macromolecules. 2009;42(5):14331438.

[18] Esteves ACC, Brokken-Zijp J, Lavèn J, Huinink HP, Reuvers NJW, Van MP, et al. Garnet particles effect on the cross-linking of PDMS and the network structures formed. Polymer. 2010;51(1):136-145.

[19] Hilding J, Grulke EA, George Zhang Z, Lockwood F. Dispersion of Carbon Nanotubes in Liquids. J Disper Sci Technol. 2003;24(1):1-41.

[20] Ahir SV, Huang YY, Terentjev EM. Polymers with aligned carbon nanotubes: Active composite materials. Polymer. 2008;49(18):3841-3854.

[21] Lu KL, Lago RM, Chen YK, Green MLH, Harris PJF, Tsang SC. Mechanical damage of carbon nanotubes by ultrasound. Carbon. 1996;34(6):814-816.

[22] Salvetat J-P, Briggs GAD, Bonard J-M, Bacsa RR, Kulik AJ, Stöckli T, et al. Elastic and Shear Moduli of Single-Walled Carbon Nanotube Ropes. Phys Rev Lett. 1999;82(5):944-947.

[23] Walters DA, Ericson LM, Casavant MJ, Liu J, Colbert DT, Smith KA, et al. Elastic strain of freely suspended single-wall carbon nanotube ropes. Appl Phys Lett. 1999;74(25):3803-3805. 
[24] Schadler LS, Giannaris SC, Ajayan PM. Load transfer in carbon nanotube epoxy composites. Appl Phys Lett. 1998;73(26):3842-3844.

[25] Hobbie EK, Fry DJ. Rheology of concentrated carbon nanotube suspensions. J Chem Phys. 2007;126(12):124907.

[26] Knauert ST, Douglas JF, Starr FW. The Effect of Nanoparticle Shape on PolymerNanocomposite Rheology and Tensile Strength. J Polym Sci, Part B: Polym Phys. $2007 ; 45 \quad 1882-1897$

[27] Winter HH. Can the gel point of a cross-linking polymer be detected by the $\mathrm{G}^{\prime}-\mathrm{G}^{\prime \prime}$ crossover? Polymer Engineering \& Science. 1987;27(22):1698-1702.

[28] Marciniec BG, J.; Urbaniak, W.; Kornetka, Z. W. Comprehensive Handbook on Hydrosililation. Oxford, U.K: Pergamon; 1992.

[29] Owen MJ. Siloxane Polymers. Prentice Hall, Englewood Cliffs, New Jersey: S. Clarson and J. A. Semlyen; 1993.

[30] Gorshov A, Kopylov Y, Dontsov A, Khazen L. International Polymer Science \& Technology. 1986;13(26).

[31] Stein J, Lewis LN, Smith KA, Lettko KX. Mechanistic studies of platinumcatalyzed hydrosilylation. J Inorg Organomet P. 1991;1(3):325-334.

[32] Lewis LN. On the mechanism of metal colloid catalyzed hydrosilylation: proposed explanations for electronic effects and oxygen cocatalysis. J Am Chem Soc. 1990;112(16):5998-6004.

[33] Malkin AY, Kulichikhin SG. Rheokinetics: rheological transformations in synthesis and reactions of oligomers and polymers. Karlsruhe: Hüthig \& Wepf Verlag; 1996. 
[34] Ramanathan T, Liu H, Brinson LC. Functionalized SWNT/polymer nanocomposites for dramatic property improvement. J Polym Sci, Part B: Polym Phys. 2005;43(17):2269-2279. 


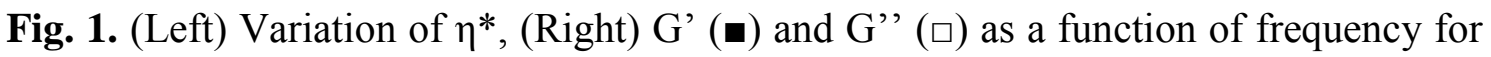
$\mathrm{MWNT} / \equiv \mathrm{SiH}$ suspensions at different dispersion times.

Fig. 2. Optical micrographs of 0.5 wt. $\%$ MWNT/PDMS suspensions after shearing 2,4 and 24 hours.

Fig. 3. Loss tangent $\tan (\delta)$ as a function of the dispersion time employed for $\mathrm{MWNT} / \equiv \mathrm{SiH}$ suspensions. The inset shows a close up for the sake of clarity.

Fig. 4. Schematic representation of the physical liquid-solid transition in $\mathrm{MWNT} / \equiv \mathrm{SiH}$ suspensions.

Fig. 5. SEM images (micrographs at $x 2.500$ magnification and inset micrographs at x10.000 magnification) of cross-linked MWNT/PDMS nanocomposites (using the suspensions previously prepared with 2 and $24 \mathrm{~h}$ dispersion time).

Fig. 6. Course of the cross-linking reaction for PDMS control sample.

Fig. 7. (Left) $\mathrm{C}(1 \mathrm{~s})$ and (right) $\mathrm{O}(1 \mathrm{~s})$ XPS spectra of pristine MWNTs employed in this work.

Fig. 8 (Left) Dynamic time sweep for the control sample and MWNT/PDMS samples. (Right) $G^{\prime}$ and $G^{\prime \prime}$ crossover point as a function of the dispersion time.

Fig. 9. Rheological conversion degree $\beta$ vs. cross-linking time for the different MWNT/PDMS nanocomposites. Experimental (dots) and theoretical data (solid lines) for a first order rheo-kinetic expression are displayed. 
Fig. 1
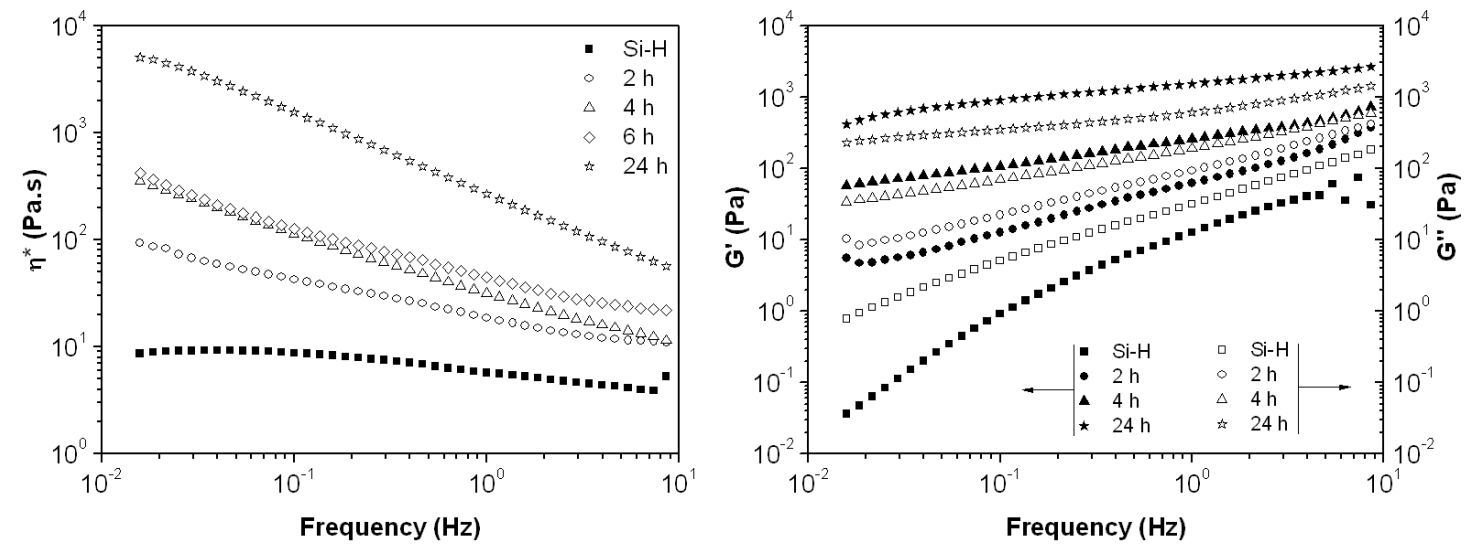
Fig. 2
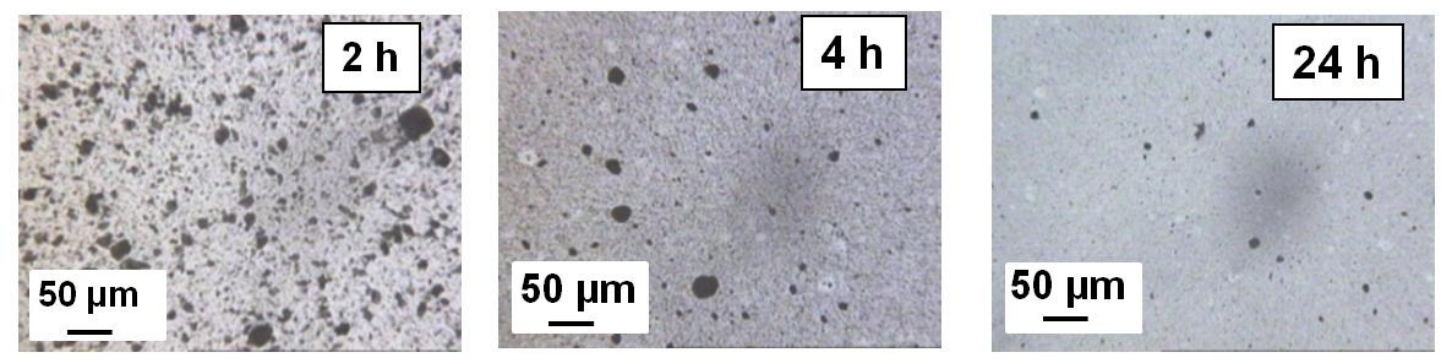
Fig. 3

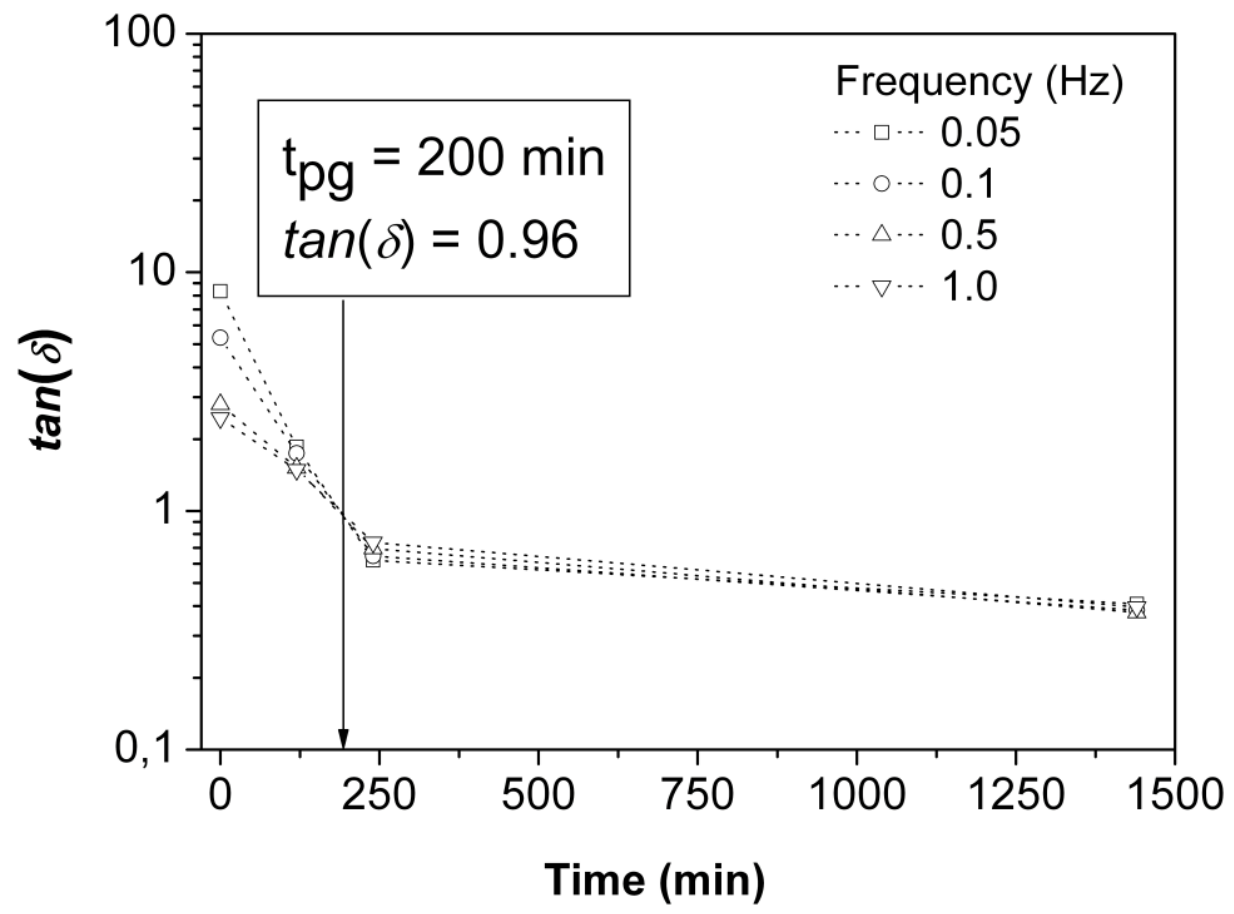


Fig. 4
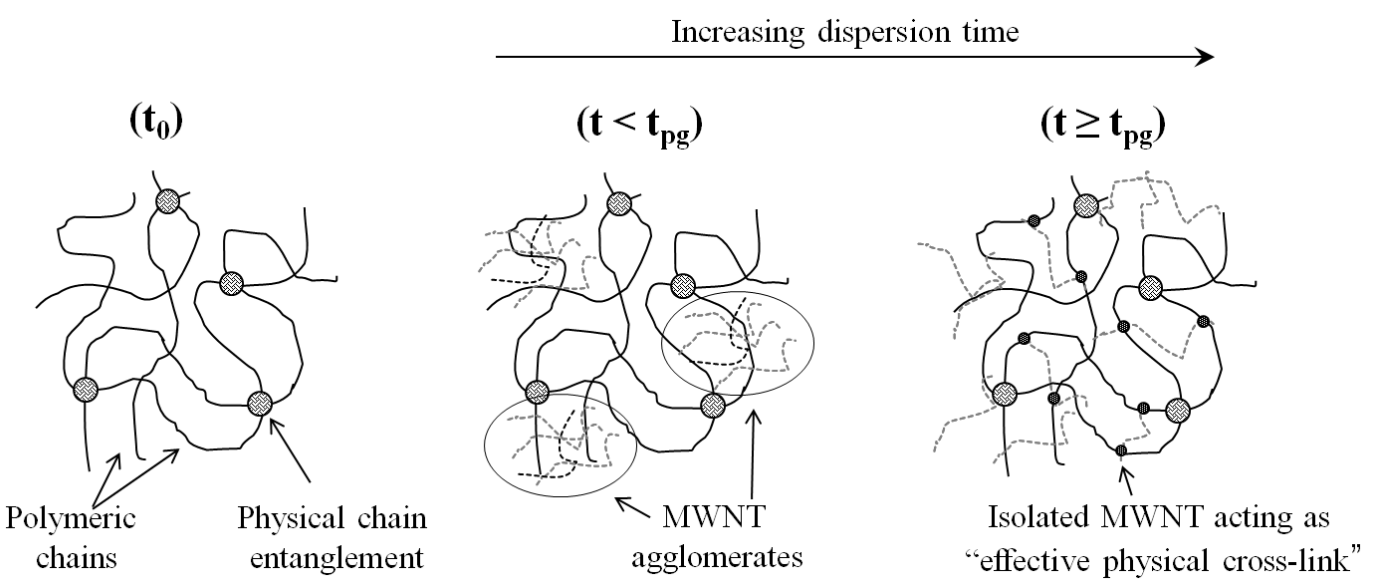
Fig. 5
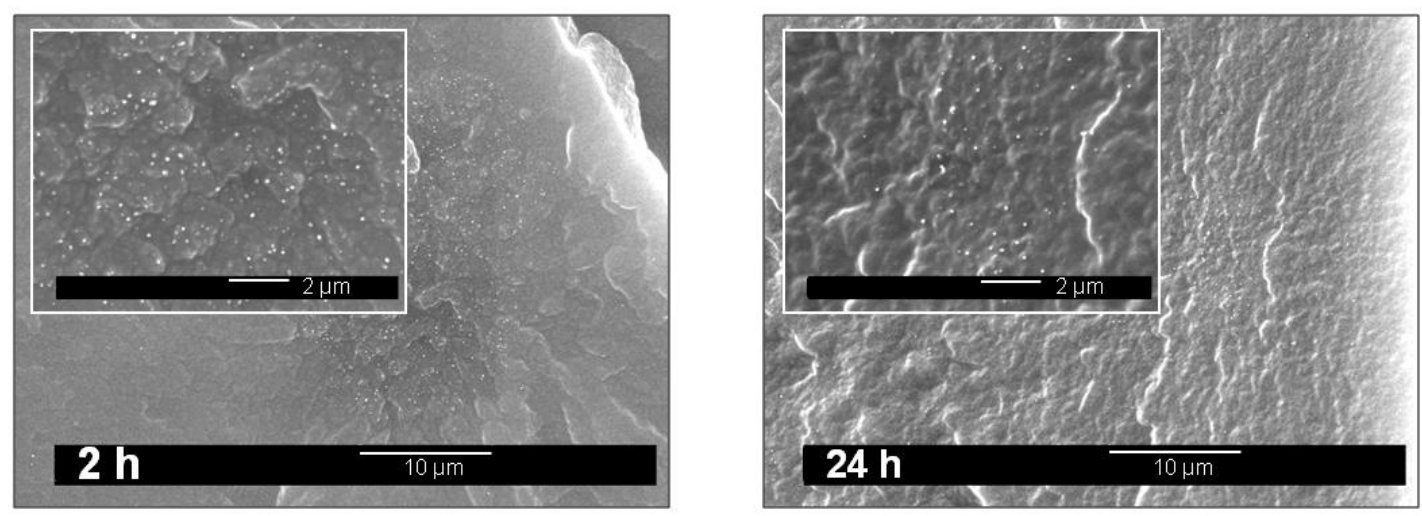
Fig. 6

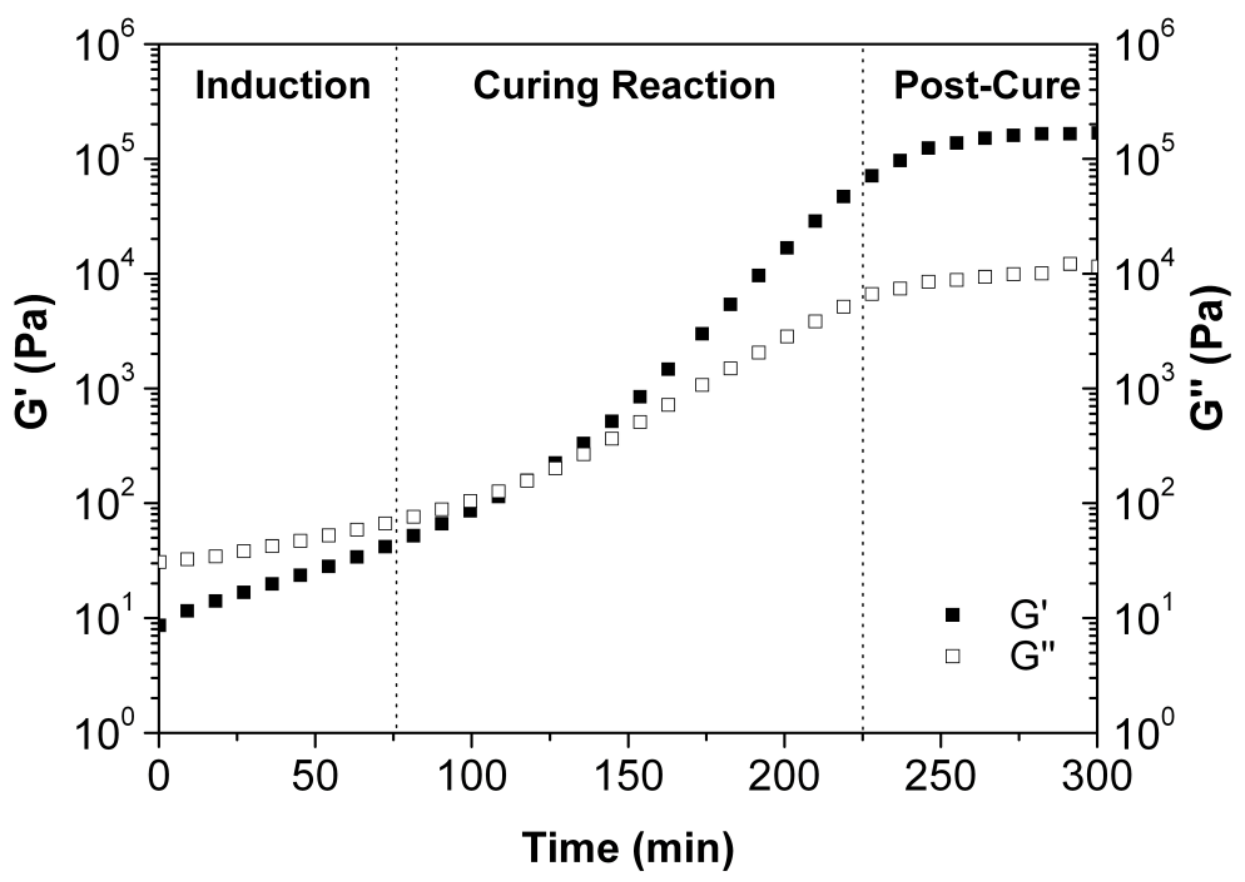


Fig. 7
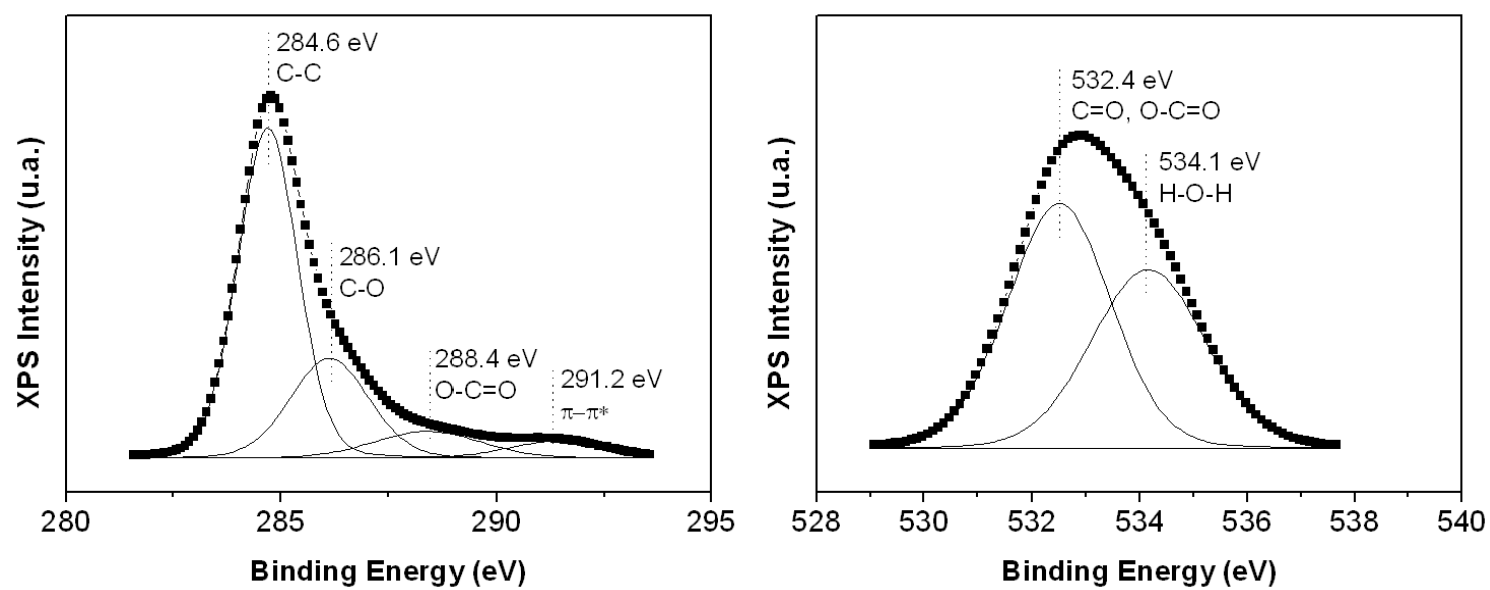
Fig. 8
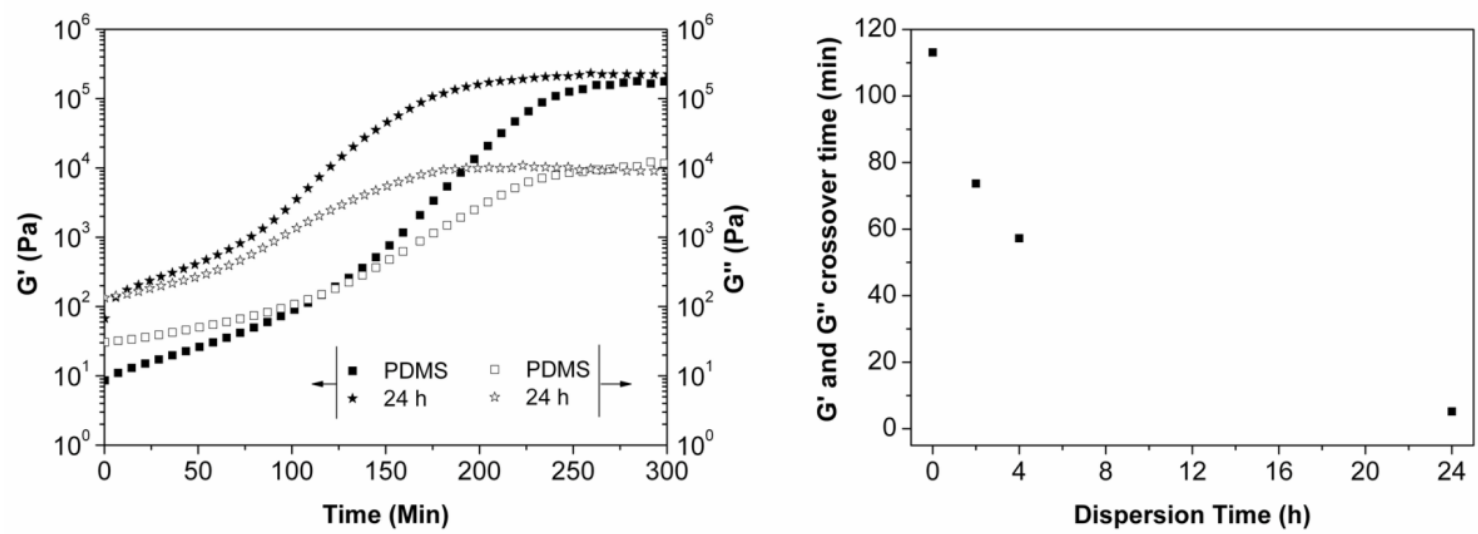
Fig. 9

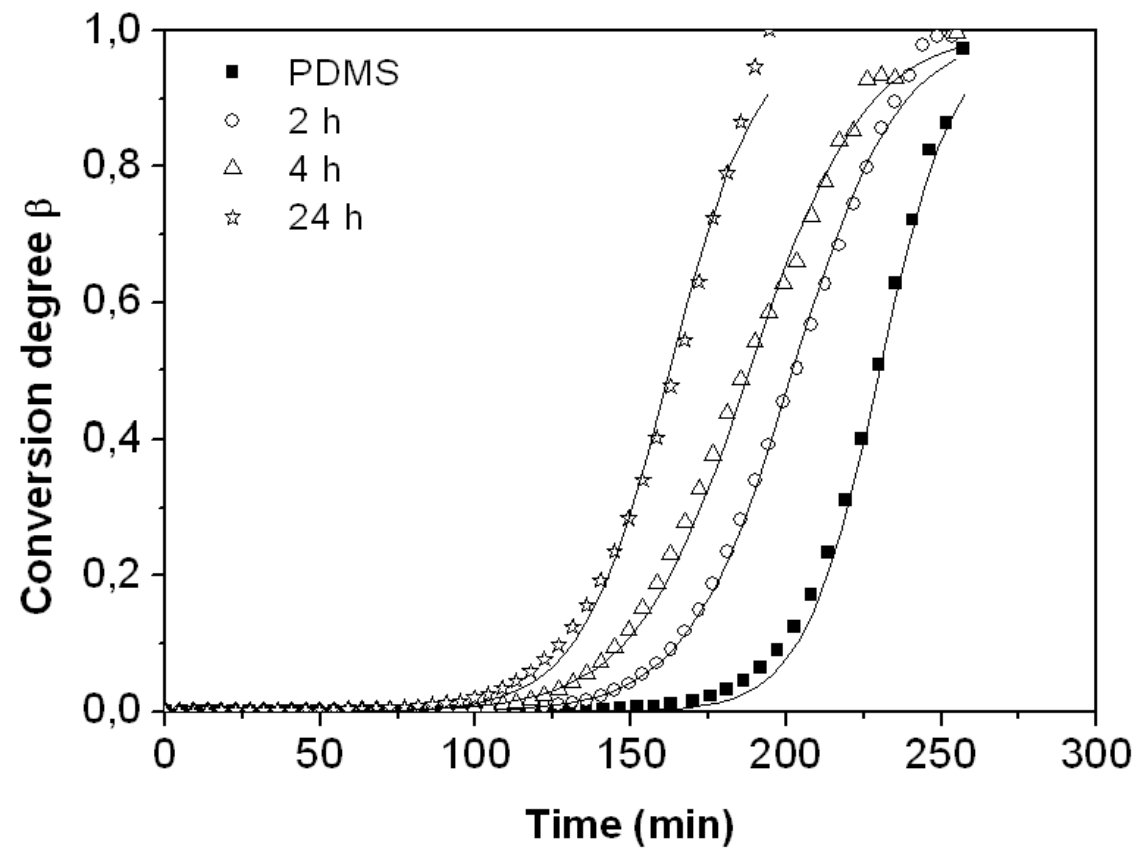


Table 1. Rheo-kinetic parameters associated with first-order equation

\begin{tabular}{cccc}
\hline \multirow{2}{*}{ Sample } & \multicolumn{2}{c}{ Rheo-kinetic parameters } & \multicolumn{2}{c}{ Correlation coefficient } \\
\cline { 2 - 4 } & $k\left(\mathrm{~min}^{-1}\right)$ & $c$ & $r^{2}$ \\
\hline PDMS & $4.72 \times 10^{-10}$ & $0.17 \times 10^{9}$ & 0.997 \\
MWNT/PDMS & & & \\
$2 \mathrm{~h}$ & $3.56 \times 10^{-7}$ & $0.16 \times 10^{6}$ & 0.998 \\
$4 \mathrm{~h}$ & $2.02 \times 10^{-6}$ & $0.02 \times 10^{6}$ & 0.995 \\
$24 \mathrm{~h}$ & $7.56 \times 10^{-5}$ & $0.09 \times 10^{6}$ & 0.995 \\
\hline
\end{tabular}


Table 2. Values of $G^{\prime}$ at the post-cure regimen and average molecular weight of chains between cross-links, $M_{c}$, for PDMS and its MWNT nanocomposites

\begin{tabular}{ccc}
\hline Sample & $G^{\prime}(\mathrm{Pa})$ at $300 \mathrm{~min}$. & $\mathrm{M}_{\mathrm{c}}(\mathrm{g} / \mathrm{mol})$ \\
\hline PDMS & 159000 & $5847 \pm 485$ \\
MWNT /PDMS & & \\
$2 \mathrm{~h}$ & 156000 & $4578 \pm 371$ \\
$4 \mathrm{~h}$ & 168000 & $4603 \pm 346$ \\
$24 \mathrm{~h}$ & 224800 & $4082 \pm 105$ \\
\hline
\end{tabular}

